\title{
Neural correlates of syntax production
}

\section{in schizophrenia}

\author{
TILO T. J. KIRCHER, TOMASINA M. OH, MICHAEL J. BR AMMER \\ and PHILIP K. McGUIRE
}

\section{Background The production of grammatically complex sentences is impaired in schizophrenia. It has been suggested that impaired syntax processing reflects a risk for the disorder.}

Aims To examine the neural correlates of syntax production in people with schizophrenia using functional magnetic resonance imaging ( $\mathrm{FMRI}$ ).

Method Sixpatients with schizophrenia and six healthy volunteers spoke about seven Rorschach inkblots for 3 min each while correlates of brain activation were measured with $\mathrm{fMRI}$. Participants produced varying amounts of syntactically simple and complex sentences during each 3 min run. The number of simple and complex sentences was correlated separately with the BOLD contrast.

Results In the comparison between the control group and the patient group, the number of complex sentences produced was correlated with activation in the posterior portion of the right middle temporal (Brodmann area 2I) and left superior frontal (BA I0) gyri in the control group but not in the patients.

Conclusions The absence of activation in the right posterior temporal and left superior frontal cortex in patients with schizophrenia might contribute to the articulation of grammatically more simple speech in people with this disorder.

Declaration of interest None.
Syntax refers to the way that words are combined grammatically to form meaningful phrases or sentences. Relative to healthy controls, patients with schizophrenia have deficits with both comprehension (Bagner et al, 2003) and production (Thomas et al, 1996b; Oh et al, 2002) of syntactically complex sentences. These deficits are especially evident in patients with formal thought disorder (Rodriguez-Ferrera et al, 2001) and do not seem to be simply a function of impaired semantic processing or general intellectual functioning (Condray et al, 2002). It has been proposed that impaired syntax processing reflects a risk for the disorder (DeLisi, 2001).

In this study we examined the neural correlates of syntax generation on a sentence level, using functional magnetic resonance imaging (fMRI) to observe participants articulating grammatically complex sentences. As syntactic processing in healthy individuals involves the inferior frontal and lateral temporal cortices (Kaan $\&$ Swaab, 2002), we predicted that the production of complex sentences would be correlated with activation in these areas in the control group, and that patients with schizophrenia would exhibit attenuated engagement of the left inferior frontal cortex and both left and right lateral temporal cortex.

\section{METHOD}

In the present investigation we used data derived from an earlier study: full details of the participants, the stimuli used, speech recording, $\mathrm{fMRI}$ and analysis are given by Kircher et al (2001b, 2002). The patient group comprised six right-handed men with a DSM-IV diagnosis of schizophrenia (American Psychiatric Association, 1994), stabilised on neuroleptic medication, with prominent positive formal thought disorder. The control group consisted of six healthy individuals who were matched for age, gender, verbal IQ, immediate memory recall, attention and socio-demographic variables; see Kircher et al (2002) for details.

During scanning, seven Rorschach inkblot plates were presented consecutively on a screen. Participants were asked to speak about whatever came to mind on viewing the inkblot, starting as soon as the stimulus appeared; they spoke freely and no prompting was given if they paused or stopped. Each of the seven plates was presented for 3 min (one 'run') with breaks of about $1 \mathrm{~min}$ between each presentation. The participants' speech during scanning was recorded on a computer.

\section{Linguistic analysis}

Acoustic noise generated by image acquisition was filtered from the recordings of the participants' speech, which was transcribed verbatim from these recordings and subsequently analysed from the transcripts. The number of simple sentences (e.g. 'I see a moth') and sentences with subordination (e.g. 'I see a moth that is blue') were evaluated using Brief Syntactic Analysis (Thomas et al, 1996a) by one of the authors (T.O.), who was masked to the speaker's identity. Brief Syntactic Analysis is a linguistic analysis that rates spontaneous speech for syntactic complexity. In this analysis, sentences were classified as one of the following:

(a) Simple sentences: sentences consisting of a subject and predicate, and containing one finite verb, e.g. 'It looks like a brain'.

(b) Complex sentences: sentences consisting of one independent clause together with one or more subordinate (embedded) clauses (e.g. 'I jumped at the sound of his voice, which was inordinately loud in the silence'). In such sentences the relationship between the independent clause and its subordinate clauses is a hierarchical one; a complex sentence may therefore have one or more levels of embedding, depending on the relationship between the subordinate clauses and the main one.

Each 3 min scanning run was broken down into nine epochs of $20 \mathrm{~s}$, and a total score for simple and complex sentences was obtained for each epoch. 


\section{Image acquisition and data analysis}

The method of image acquisition and data analysis has been fully described by Kircher et al (2002). Gradient-echo echoplanar MR images were acquired using a $1.5 \mathrm{~T}$ GE Signa System (General Electric, Milwaukee, Wisconsin, USA): 14 planes, $60 T_{2}{ }^{*}$ weighted MR images; time to echo (TE) $40 \mathrm{~ms}$, repetition time (TR) $3000 \mathrm{~ms}$, $\theta=90^{\circ}$, in-plane resolution $3.1 \mathrm{~mm}$, slice thickness $7 \mathrm{~mm}$, slice skip $0.7 \mathrm{~mm}$.

For fMRI data analysis (Brammer et al, 1997), the behavioural data (number of simple and complex sentences per $20 \mathrm{~s}$ epoch in the two runs per individual with the highest variance and highest number of maxima and minima) were interpolated using a cubic spline to obtain a behavioural value corresponding to each fMRI volume acquired (one value per TR). The dependent variable (observed time series at each voxel) was regressed on the independent variable (number of simple and complex sentences). Further analysis was performed as described by Kircher et al (2002). For group comparisons, we computed a mask composed of all voxels activated at a stringent voxel-wise threshold $(0.001)$ in either of the two groups or conditions that we needed to compare. We then carried out between-condition and between-group comparisons within this masked region to reduce the multiple comparison problem and thus enhance sensitivity. Comparison of groups was performed by computing the difference between the mean levels of activation at each voxel in standard space over all participants in each group. The significance of this difference was then assessed by reference to a null distribution obtained by repeated permutation of group membership and recomputation of the mean difference.

\section{RESULTS}

\section{Behavioural measures}

In the two Rorschach plates per individual used for the fMRI analysis, the number of simple sentences spoken by the patients ranged from 3 to 26 (mean 13.8, s.d.=7.4); in the volunteers the range was 2-28 (mean $14.2, \quad$ s.d. $=8.2 ; \quad P=0.9$, Mann-Whitney $U=71.5$ ). The number of complex sentences spoken by the patients ranged from 5 to 13 (mean 8.5, s.d. $=2.8$ ), whereas in the volunteers the range was 6-20 (mean 13.5, s.d. $=4.1 ; \quad P=0.003$, $U=21.0)$. There was no correlation

Table I Main foci of signal changes in the control group during production of continuous speech: correlations with simple and complex sentences are shown $(P<0.001)$, followed by statistical differences between the two conditions $(P<0.05)$

\begin{tabular}{|c|c|c|c|c|c|c|}
\hline \multirow[t]{2}{*}{ Region } & \multirow[t]{2}{*}{ BA $^{\prime}$} & \multirow[t]{2}{*}{ Hemisphere } & \multicolumn{3}{|c|}{ Coordinates $(\mathrm{mm})^{\prime}$} & \multirow{2}{*}{$\begin{array}{c}\text { Voxels } \\
\text { (n) }\end{array}$} \\
\hline & & & $x$ & $y$ & z & \\
\hline \multicolumn{7}{|l|}{ Simple sentences } \\
\hline Thalamus & & $\mathrm{L}$ & -5 & -8 & I & 7 \\
\hline Primary visual cortex & $18 / 19$ & $L$ & -30 & -84 & 8 & 6 \\
\hline \multicolumn{7}{|l|}{ Complex sentences } \\
\hline Superior temporal sulcus & $21 / 22$ & $\mathbf{R}$ & 42 & -47 & 8 & 12 \\
\hline Posterior middle temporal gyrus & 39 & $\mathrm{~L}$ & -49 & -63 & 17 & 8 \\
\hline Superior frontal gyrus & 10 & $\mathrm{~L}$ & -19 & 50 & 15 & II \\
\hline Cingulate gyrus & 25 & $\mathbf{R}$ & 9 & 7 & -7 & 9 \\
\hline Caudate nucleus & & $\mathbf{R}$ & -9 & 7 & -7 & 9 \\
\hline \multicolumn{7}{|l|}{ ANOVA } \\
\hline \multicolumn{7}{|l|}{ Simple sentences $>$ complex sentences } \\
\hline Middle occipital gyrus & 19 & $\mathrm{~L}$ & -29 & -83 & 9 & 7 \\
\hline \multicolumn{7}{|l|}{ Complex sentences $>$ simple sentences } \\
\hline Caudate nucleus & & $\mathbf{R}$ & -9 & 7 & -7 & 8 \\
\hline Superior temporal sulcus & $21 / 22$ & $\mathbf{R}$ & 41 & -46 & 9 & 6 \\
\hline Superior frontal gyrus & 10 & $\mathrm{~L}$ & -9 & 56 & 9 & 4 \\
\hline
\end{tabular}

ANOVA, analysis of variance; BA, Brodmann area; L, left; R, right.

I. See Talairach \& Tournoux (1988)

between the number of complex and simple sentences produced in either group. The groups did not differ in the total number of sentences produced (controls: median 31.5, maximum 36.0; patients: median 29.5, maximum 39.0; $U=71.0, P=0.9$ ).

\section{Head movement}

Analysis of the estimated time series of displacements in three dimensions revealed no evidence of major head motion in any individual. The maximum amount of head movement in the three dimensions $x, y$ and $z$ in the runs per patient was $x=0.5 \mathrm{~mm} \quad$ (s.d. $=0.3$ ), $y=0.7 \mathrm{~mm} \quad$ (s.d. $=$ $0.3), z=1.4 \mathrm{~mm} \quad(\mathrm{~s} . \mathrm{d} .=1.6)$ and in the controls it was $x=0.4 \mathrm{~mm} \quad$ (s.d. $=0.3$ ), $y=0.3 \mathrm{~mm} \quad($ s.d. $=0.4) \quad$ and $\quad z=0.8 \mathrm{~mm}$ (s.d. $=0.8$ ).

\section{Cerebral activation}

\section{Control group}

In the control group the number of simple sentences produced per $20 \mathrm{~s}$ period was positively correlated $(P<0.001)$ with the BOLD response in the left thalamus and primary visual cortex. The main correlations for complex sentences $(P<0.001)$ were with responses in the posterior part of the left middle temporal gyrus, Brodmann area (BA) 39, and in the posterior portion of the right superior temporal sulcus, BA 21/22 (Table 1). Between-condition differences in median correlation coefficient were examined using analysis of variance (ANOVA) at each voxel with probability of type I error at $P<0.05$. Significantly stronger correlations for complex $v$. simple sentences were evident in the right posterior superior temporal sulcus (BA 21) and the left superior frontal gyrus (BA 10; Table 1).

\section{Patient group}

In the patient group the number of simple sentences produced per $20 \mathrm{~s}$ period was positively correlated $(P<0.001)$ with the BOLD response in the cerebellum bilaterally (vermal lobule VI; Schmahmann et al, 1999). The main correlations for complex sentences $(P<0.001)$ were with responses in the middle temporal gyri bilaterally (BA 21), in a part anterior and inferior to that activated in the control group. Between-condition differences in median correlation coefficient were examined using an ANOVA at each voxel. This indicated 
that there were significantly stronger correlations for complex sentences than for simple sentences with responses in the right middle temporal gyrus (BA 21; $P<0.05$ ), again in a region anterior and inferior to the site of the analogous differences in the control group (Table 2; Fig. 1).

\section{Differences in activations for simple sentences} between groups

Signal changes correlating with simple sentences were compared between groups $(P<0.05)$. Participants in the control group activated the left primary visual cortex and the thalamus more than the patients, whereas those in the patient group engaged the right cerebellar cortex more than the controls (Table 3).

\section{Differences in activations for complex} sentences between groups

Signal changes correlating with complex sentences were compared between groups $(P<0.05)$. The control group activated the right posterior middle temporal gyrus (BA 21) and the supramarginal gyrus (BA 40) more than the patients, whereas the patient group engaged the left fusiform/parahippocampal gyrus more than the controls (Table 4, Fig. 1).

\section{DISCUSSION}

In this study, participants spoke continuously about Rorschach inkblots while their regional brain activity was being measured with fMRI. In accordance with previous behavioural studies on syntax production in schizophrenia, we found that the patient group produced fewer complex sentences than controls (Thomas et al, 1996b; Oh et $a l, 2002)$. However, the numbers of simple sentences produced by the two groups were similar.

\section{Syntax production in the control group}

The rate of production of each sentence type was correlated with the BOLD response in each group. In members of the control group there was a positive correlation between the number of complex sentences and activation in the left posterior middle temporal gyrus, which is part of Wernicke's area, and in the right superior temporal sulcus. Both these areas have consistently been activated in functional imaging studies of syntax comprehension in
Table 2 Main foci of signal changes in participants with schizophrenia during production of continuous speech: correlations with simple and complex sentences are shown $(P<0.00 \mathrm{I})$, followed by statistical differences between the two conditions $(P<0.05)$

\begin{tabular}{lllll}
\hline Region & BA Hemisphere & \multicolumn{2}{c}{ Coordinates (mm) } & Voxels \\
\cline { 3 - 4 } & & $x$ & $y$ & (n)
\end{tabular}

\begin{tabular}{|c|c|c|c|c|c|c|}
\hline \multicolumn{7}{|l|}{ Simple sentences } \\
\hline \multirow[t]{2}{*}{ Cerebellum } & & $\mathrm{L}$ & -15 & -57 & -29 & 37 \\
\hline & & $\mathbf{R}$ & 10 & -53 & -31 & 17 \\
\hline \multicolumn{7}{|l|}{ Complex sentences } \\
\hline Hippocampal area & & $L$ & -35 & -29 & $-I I$ & 19 \\
\hline \multirow[t]{2}{*}{ Middle temporal gyrus } & 21 & $\mathbf{R}$ & 54 & -28 & -6 & 15 \\
\hline & & $\mathrm{L}$ & -48 & -40 & -10 & 6 \\
\hline Precentral gyrus & 6 & $\mathbf{R}$ & 46 & -10 & 37 & 14 \\
\hline Primary visual cortex & $18 / 19$ & $\mathrm{~L}$ & -31 & -71 & 9 & II \\
\hline Inferior frontal gyrus & 46 & $\mathrm{~L}$ & -40 & 37 & 9 & 7 \\
\hline Posterior insula & & $\mathrm{L}$ & -26 & -15 & 15 & 7 \\
\hline \multicolumn{7}{|c|}{ ANOVA } \\
\hline \multicolumn{7}{|c|}{ Simple sentences $>$ complex sentences } \\
\hline Cerebellum & & $L$ & -17 & -50 & -29 & 29 \\
\hline \multicolumn{7}{|c|}{ Complex sentences $>$ simple sentences } \\
\hline Postcentral gyrus & $1 / 3$ & $\mathbf{R}$ & 49 & -14 & 37 & 9 \\
\hline Primary visual cortex & 19 & $\mathbf{R}$ & -32 & -69 & 9 & 6 \\
\hline Middle temporal gyrus & 21 & $\mathbf{R}$ & 52 & -33 & -7 & 4 \\
\hline
\end{tabular}

ANOVA, analysis of variance; BA, Brodmann area; L, left; $R$, right.

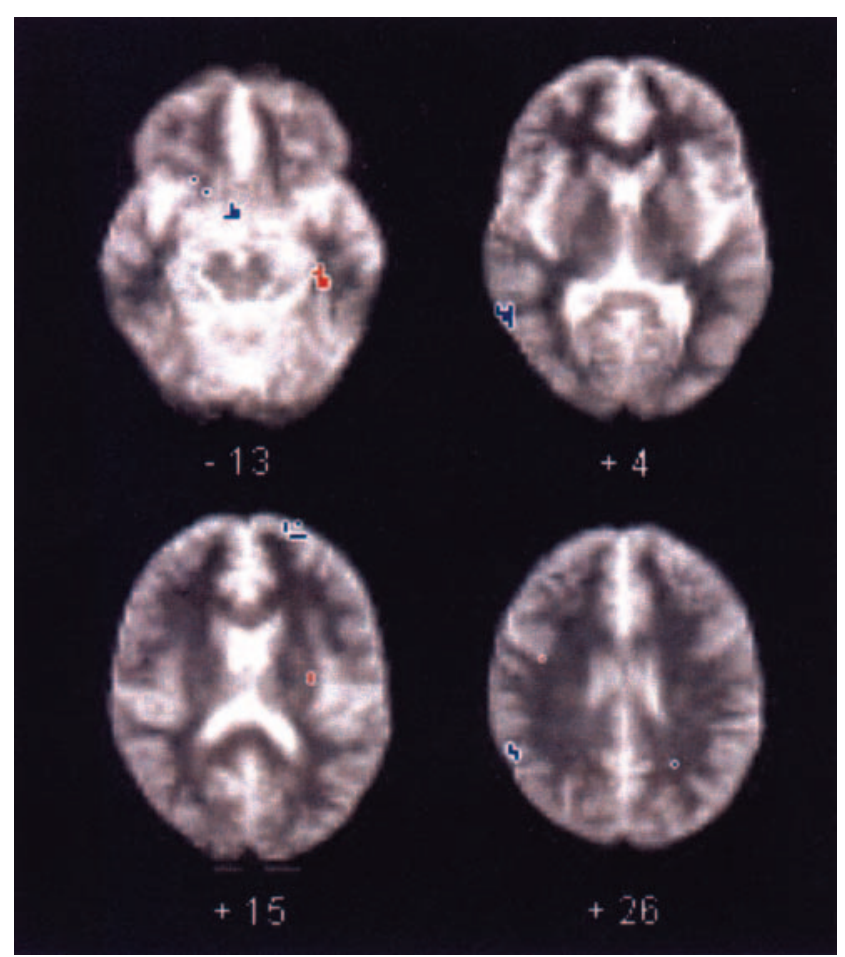

Fig. I Brain activation maps: functional magnetic resonance imaging signal changes correlated with the amount of syntactically complex sentences produced per 20 s epoch across two 3 min runs, showing significant differences in power of response between the patient and control groups $(P<0.05)$. Blue voxels indicate greater power of response in the control participants (right posterior middle temporal and left superior front gyrus, right nucleus accumbens); red voxels indicate greater power of response in the participants with schizophrenia (left parahippocampal gyrus). The left side of the brain is shown on the right side of the image; Talairach $z$ coordinates (Talairach \& Tournoux, 1988) are given below each slice. 
Table 3 Differences in activation $(P<0.05)$ for simple sentences between patient and control groups

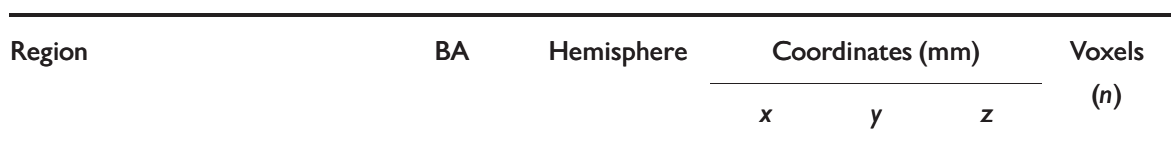

\begin{tabular}{|c|c|c|c|c|c|c|}
\hline \multicolumn{7}{|c|}{ Simple sentences: controls $>$ patients } \\
\hline Primary visual cortex & $18 / 19$ & $\mathrm{~L}$ & -29 & -83 & 9 & 7 \\
\hline Thalamus & & $\mathrm{L}$ & -3 & -6 & -2 & 5 \\
\hline \multicolumn{7}{|c|}{ Simple sentences: patients $>$ controls } \\
\hline Cerebellum & & $\mathrm{R}$ & 6 & -47 & -18 & 10 \\
\hline
\end{tabular}

BA, Brodmann area; L, left; $R$, right.

Table 4 Differences in activation $(P<0.05)$ for complex sentences between patient and control groups

\begin{tabular}{|c|c|c|c|c|c|c|}
\hline \multirow[t]{2}{*}{ Region } & \multirow[t]{2}{*}{ BA } & \multirow[t]{2}{*}{ Hemisphere } & \multicolumn{3}{|c|}{ Coordinates $(\mathrm{mm})$} & \multirow{2}{*}{$\begin{array}{c}\text { Voxels } \\
\text { (n) }\end{array}$} \\
\hline & & & $x$ & $y$ & $\mathbf{z}$ & \\
\hline \multicolumn{7}{|l|}{ Complex sentences: controls $>$ patients } \\
\hline Posterior middle temporal gyrus & 21 & $\mathbf{R}$ & 58 & -50 & 4 & 8 \\
\hline \multirow[t]{2}{*}{ Nucleus accumbens } & & $\mathbf{R}$ & 9 & 6 & -7 & 6 \\
\hline & & $\mathbf{R}$ & 6 & 3 & -13 & 6 \\
\hline Superior frontal gyrus & 10 & $L$ & -14 & 56 & 15 & 5 \\
\hline Supramarginal gyrus & 40 & $\mathrm{R}$ & 52 & -44 & 26 & 4 \\
\hline \multicolumn{7}{|c|}{ Complex sentences: patients > controls } \\
\hline Fusiform/parahippocampal gyrus & $35 / 36$ & $\mathrm{~L}$ & -35 & -25 & -13 & 7 \\
\hline
\end{tabular}

BA, Brodmann area; L, left; R, right.

healthy volunteers (Kaan \& Swaab, 2002). However, whereas previous studies investigated syntax in the context of sentence comprehension, the neural correlates in our study were those of syntactic production during continuous speech. We thus interpret the signal changes in the left middle temporal gyrus and right superior temporal sulcus as being related to the production of syntactically complex sentences.

\section{Syntax production in the patient group}

In the patient group the production of complex sentences was also correlated with bilateral activation in the temporal cortex, but in regions anterior and inferior to those engaged in the controls. Direct comparison of the correlations in the two groups revealed significantly greater activation in the right posterior middle temporal gyrus, right supramarginal gyrus and left superior frontal gyrus in the control group compared with the patients. This weaker engagement of the posterior temporal cortex and adjacent inferior parietal lobule may be related to the impairment in producing complex sentences that we observed in the patients at the behavioural level. However, we cannot entirely exclude the possibility that these functional differences were related to semantic processing. Complex sentences are more complicated than simple sentences in terms of syntax as well as their semantic content. Reading sentences involves the temporal cortex (Kuperberg et al, 2000), and patients with schizophrenia show deficits in semantic processing at the behavioural level (Bagner et al, 2003). responsible for the temporal activations, we would also expect at least small signal changes in these areas for the correlations with simple sentences. An increased number of simple sentences per unit of time also conveys increased semantic content. We did not find such a correlation in either group and would therefore suggest that, in line with recent research (Kaan \& Swaab, 2002), the temporal activations reflect syntactic complexity.

The impaired posterior temporal activation we observed in the patients is in line with other data demonstrating structural and functional abnormalities in this area in schizophrenia. Thus, the production of thought-disordered speech in another study However, if semantic content were solely of the same patients correlated negatively with signal changes in the left superior temporal gyrus (Kircher et al, 2001b), and lateralisation changes in the same areas have been described during a sentence completion task, also in the same sample (Kircher et al, 2001a). The temporal cortices have been implicated in the generation of auditory verbal hallucinations (Dierks et al, 1999; Shergill et al, 2000). Structural imaging studies (for review, see Shenton et al, 2001) have described volumetric anomalies in the lateral temporal gyri in schizophrenia, particularly in patients with formal thought disorder. Our findings are also consistent with abnormalities in the planum temporale during simple acoustic processing, where abnormalities in lateralisation patterns have been described (Rockstroh et al, 1998; Kircher et al, 2004).

\section{Frontal lobe}

Apart from the temporal cortex, the only other region that was significantly more correlated with complex rather than simple sentence production in the control group was in the left prefrontal cortex, close to the frontal pole (BA 10). This difference was not evident in the patient group and direct contrast of the correlation maps for complex sentences in the two groups revealed significantly greater activation in the controls than in the patients. Other studies have found activation in the proximity of this region during syntax comprehension in healthy volunteers (Newman et al, 2001). Although we predicted impaired differential engagement of the left inferior frontal gyrus rather than this more anterior region in the two groups, these observations suggest that this might also be related to impaired syntactic processing in people with schizophrenia.

It has been proposed that Broca's area is involved in syntactic processing only when the demands on verbal working memory are high (Kaan \& Swaab, 2002). Although the articulation of syntactically complex sentences might have engaged verbal working memory to a greater extent than simple sentences, the difference in the memory load might have been too small to lead to differential left inferior frontal activation. The absence of correlations between the production of complex sentences and the engagement of motor-related areas more generally may seem surprising, given that the participants were articulating speech; however, as they were speaking at 
a rate that was self-paced and felt natural for them, the demands on articulatory processing might have been relatively constant. Activation in motor areas might thus have been at ceiling and not have shown a measurable response to fluctuations in the amount of speech produced in association with the production of complex sentences (Kircher et al, 2002).

\section{Methodological considerations}

Although overt speech can be associated with artefacts in fMRI studies secondary to articulation-related head movement (Bullmore et al, 1999), we quantified head movement during image acquisition, and found it to be minimal in both groups. Artefacts can also be introduced by air volume changes in the pharynx during phonation. However, when scanning at $1.5 \mathrm{~T}$ these effects are likely to be small, except in areas close to the orbital frontal cortex (Barch et al, 1999). Functional MRI is also associated with significant scanner noise, but all our participants reported that they were able to hear themselves speak during the task.

\section{Concluding remarks}

Our data implicate the left posterior superior temporal sulcus (Wernicke's area), the right posterior middle temporal gyrus and the left prefrontal cortex in the production of syntactically complex sentences in healthy individuals. Patients with schizophrenia failed to show this pattern of activation, with significantly weaker engagement of the right temporal and left prefrontal cortex, which may underlie the reduced production of grammatically complex sentences in these patients. Language abnormalities seem to be the only symptom that is genetically transmitted among relatives of people with schizophrenia (Cardno et al, 2001). The way in which language, genes, the brain and schizophrenia relate to each other is a very intriguing question (Crow, 2000) and needs further investigation.

\section{ACKNOWLEDGEMENTS}

T.K. was supported by the German Research Council.

\section{REFERENCES}

American Psychiatric Association (1994) Diagnostic and Statistical Manual of Mental Disorders (4th edn) (DSM-IV). Washington, DC: APA.

\section{CLINICAL IMPLICATIONS}

Patients with schizophrenia failed to activate the right temporal and left prefrontal cortex during production of grammatically complex sentences.

- Abnormalities in the superior temporal gyrus, which is a key region in the neuropathology of schizophrenia, may underlie the reduced production of syntactically complex structures in these patients.

- Impaired syntactic processing may be a risk factor in the development of schizophrenia.

\section{LIMITATIONS}

- The syntactic measures used in our study could be complemented by a more finegrained linguistic analysis.

- Because the study did not include a group of participants with schizophrenia who were not prone to formal thought disorder, it is not possible to separate the effect of schizophrenia in general from the specific propensity to produce formal thought disorder.

- The study would have benefited from the inclusion of an unmedicated patient group and a greater sample size.

TILOT. J. KIRCHER, Department of Psychiatry, University of Aachen and Tübingen, Germany and Section of Neuroimaging, Institute of Psychiatry and Guy's, King's and St Thomas' School of Medicine, London, UK; TOMASINA M. OH, Department of English Language and Literature, National University of Singapore; MICHAEL J. BRAMMER, PHILIP K. McGUIRE, Section of Neuroimaging, Institute of Psychiatry and Guy's, King's and St Thomas' School of Medicine, London, UK

Correspondence: DrT. Kircher, Department of Psychiatry, University of Aachen, Pauwelstrasse 30, D-52076 Aachen, Germany. Tel: +49 (0)24I 808 9640; fax: +49 (0)24I 808 2401 ; e-mail: tkircher@ukaachen.de

(First received 17 September 2003, final revision 5 March 2004, accepted 26 August 2004)

Bagner, D. M., Melinder, M. R. \& Barch, D. M. (2003) Language comprehension and working memory language comprehension and working memory deficits in patients with schizophrenia. Schizophrenia Research, 60, 299-309.

Barch, D. M., Sabb, F. W., Carter, C. S., et al (1999) Overt verbal responding during $\mathrm{fMRI}$ scanning: empirical investigations of problems and potentia solutions. Neuroimage, 10, 642-657.

Brammer, M. J., Bullmore, E.T., Simmons, A., et al (1997) Generic brain activation mapping in functional magnetic resonance imaging: a nonparametric approach. Magnetic Resonance Imaging, 15, 763-770.

Bullmore, E.T., Brammer, M. J., Rabe Hesketh, S., et al (1999) Methods for diagnosis and treatment of stimulus-correlated motion in generic brain activation studies using fMRI. Human Brain Mapping, 7, 38-48.

Cardno, A. G., Sham, P. C., Murray, R. M., et al (200I) Twin study of symptom dimensions in psychoses. British Journal of Psychiatry, I79, 39-45.
Condray, R., Steinhauer, S. R., van Kammen, D. P., et al (2002) The language system in schizophrenia: effects of capacity and linguistic structure. Schizophrenia Bulletin, 28, 475-490.

Crow, T.-J. (2000) Schizophrenia as the price that homo sapiens pays for language: a resolution of the central paradox in the origin of the species. Brain Research. Brain Research Reviews, 31, 118-129.

DeLisi, L. E. (200I) Speech disorder in schizophrenia: review of the literature and exploration of its relation to the uniquely human capacity for language. Schizophrenia Bulletin, 27, 48I-496.

Dierks, T., Linden, D. E., Jandl, M., et al (1999) Activation of Heschl's gyrus during auditory hallucinations. Neuron 22, 615-621.

Kaan, E. \& Swaab, T. Y. (2002) The brain circuitry of syntactic comprehension. Trends in Cognitive Science, $\mathbf{6}$, 350-356.

Kircher, T.T., Bullmore, E. T., Brammer, M. J., et al (200la) Differential activation of temporal cortex during 
sentence completion in schizophrenic patients with and without formal thought disorder. Schizophrenia Research, 50, $27-40$.

Kircher, T.T. J., Liddle, P. F., Brammer, M. J., et al (200lb) Neural correlates of formal thought disorder in schizophrenia. Archives of General Psychiatry, 58, 769774

Kircher, T. T. J., Liddle, P. F., Brammer, M. J., et al (2002) Reversed lateralisation of temporal activation during speech production in thought disordered patients with schizophrenia. Psychological Medicine, 32, 439-449.

Kircher, T. T. J., Rapp, A., Grodd, W., et al (2004) Mismatch responses in schizophrenia: a combined fMRI and whole-head MEG study. American Journal of Psychiatry, 16I, 294-304.

Kuperberg, G. R., McGuire, P. K., Bullmore, E.T., et al (2000) Common and distinct neural substrates for pragmatic, semantic, and syntactic processing of spoken sentences: an fMRI study. Journal of Cognitive Neuroscience, |2, 321-341.
Newman, A. J., Pancheva, R., Ozawa, K., et al (2001) An event-related fMRI study of syntactic and semantic violations. Journal of Psycholinguistic Research, 30, 339-364.

Oh, T. M., McCarthy, R. A. \& McKenna, P. J. (2002) Is there a schizophasia? A study applying the single case approach to formal thought disorder in schizophrenia. Neurocase, 8, 233-244.

Rockstroh, B., Clementz, B. A., Pantev, C., et a (1998) Failure of dominant left-hemispheric activation to right-ear stimulation in schizophrenia. Neuroreport, $\mathbf{9}$ 3819-3822.

Rodriguez-Ferrera, S., McCarthy, R. A. \& McKenna, P. J. (200I) Language in schizophrenia and its relationship to formal thought disorder. Psychological Medicine, 3I, 197-205.

Schmahmann, J. D., Doyon, J., McDonald, D., et al (1999) Three-dimensional MRI atlas of the human cerebellum in proportional stereotaxic space. Neuroimage, 10, 233-260.
Shenton, M. E., Dickey, C. C., Frumin, M., et al (200I) A review of MRI findings in schizophrenia. Schizophrenia Research, 49, I-52.

Shergill, S. S., Brammer, M. J., Williams, S. C. R., et a (2000) Mapping auditory hallucinations in schizophrenia using functional magnetic resonance imaging. Archives of General Psychiatry, 57, 1033-1038.

Talairach, J. \& Tournoux, P. (1988) Co-Planar Stereotaxic Atlas of the Human Brain. Stuttgart: Thieme.

Thomas, P., Kearney, G., Napier, E., et al (1996a) The reliability and characteristics of the brief syntactic analysis. British Journal of Psychiatry, 168, 334-337.

Thomas, P., Kearney, G., Napier, E., et al (1996b) Speech and language in first onset psychosis differences between people with schizophrenia, mania, and controls. British Journal of Psychiatry, 168, 337-343. 